\title{
Aprendizaje invertido: Un modelo emergente para el aprendizaje de la programación en estudiantes universitarios
}

\author{
Flipped learning: an emergent model for learning of programming in \\ college students
}

\section{alfredo Díaz Pérez}

alfredojodp@hotmail.com

Código ORCID: 0000-0002-2091-2176

\author{
Joseabel Cegarra Conde \\ joseabelcegarra@hotmail.com \\ Código 0000-0001-5395-528X
}

\author{
Luisa Serra López \\ serra_sl@yahoo.com \\ Código 0000-0002-7561-2541
}

Universidad del Zulia, Núcleo Costa Oriental del Lago, Venezuela

Artículo recibido en octubre 2020 | Arbitrado en noviembre 2020 | Publicado en enero 2021

\section{Resumen}

La presente investigación tuvo como propósito describir los pilares del aprendizaje invertido en los docentes de programación del Programa de Humanidades y Educación, mención Informática, de la Universidad del Zulia, Núcleo Costa Oriental del Lago. El estudio fue de tipo descriptivo y de campo, con diseño no experimental-transeccional. Para la recolección de datos, se aplicó un cuestionario, validado por cinco expertos. Estuvo estructurado por 12 ítems con escala tipo Likert. Se estimó la confiabilidad utilizando el coeficiente Alfa de Cronbach, resultando en 0.92, es decir, fue altamente confiable. Para el análisis de los datos se utilizaron estadísticas descriptivas, representadas por los promedios aritméticos de cada pilar: ambiente flexible, cultura de aprendizaje, contenido intencional y educadores profesionales. Se procedió a realizar la discusión de los resultados con las teorías, concluyendo que los docentes deben familiarizarse con este modelo para poder utilizarlo, pues, éstos ya poseen competencias tecnológicas bien definidas y conocen a profundidad los contenidos de sus unidades curriculares, lo cual es una ventaja para su implementación.

\footnotetext{
Abstract

The purpose of this research, was to describe the columns of flipped learning in programming teachers of humanities and education program in the University of Zulia. It was classified as descriptive-field with non-experimentaltransectional design. For data collection was applied one questionnaire. It measured the four columns of flipped learning in five programming teachers. It was validated by five experts. It was structured by 12 items with Likert-type Scale. The reliability was calculated using the Alfa-Cronbach coefficient, obtaining a value of 0.92 , In that way, it was highly reliable. For the data analysis, there were used descriptive statistics, represented by the arithmetic averages of each column: Flexible environment, Learning culture, Intentional content and Professional teachers. Then, the results discussion was made with the theories, concluding that the teachers must be familiarized with this model in order to apply, because, they already have strong technological skills and deeply know the contents of their signatures, in fact, this is an advantage for its implementation.
}

Palabras clave:

Aprendizaje invertido; modelo de aprendizaje; programación

\section{Keywords:}

Flipped learning, learning model, programming 
INTRODUCCIÓN

En el marco de las estrategias y modelos de enseñanza, han surgido paradigmas que buscan transformar la educación tradicional, dinamizando este proceso, en virtud de formar individuos integrales, de libre pensamiento; pero sobre todo, responsables de su propio aprendizaje. Además, el auge de las nuevas tecnologías de información y comunicación (TIC) ha permitido adherirlas al ámbito educativo, proporcionando herramientas que amplían el horizonte académico con recursos interactivos, llamativos e innovadores que sirven como base para consolidar habilidades explícitas en los estudiantes, haciéndolos pertinentes al contexto en el cual se desempeñarán profesionalmente.

Sobre la base de las ideas anteriores, Bergmann y Sams (2014) crearon un modelo de enseñanza-aprendizaje denominado flipped learning (o aprendizaje invertido), en el cual, la instrucción directa se realiza fuera del aula y los encuentros presenciales se utilizan para desarrollar actividades de construcción y consolidación del conocimientos. En ese sentido, se aprovecha el tiempo previo a las clases en el aula para que los estudiantes accedan recursos multimedia (videos, animaciones, presentaciones electrónicas, infografías, entre otros) desde sus hogares, sitios de trabajo o incluso lugares públicos con acceso a internet a fin de optimizar los encuentros áulicos donde se convierten en constructores de saberes y actores activos del proceso de aprendizaje.

En ese propósito, Martínez y Esquivel (2018) expresan que la idea de este modelo es promover el desarrollo de habilidades instrumentales en el estudiante a partir de la construcción de sus propios conceptos antes del encuentro dentro del aula, luego, se producen las disertaciones grupales y con el docente, haciendo las clases más interactivas y productivas para culminar con la retroalimentación de saberes donde se comparten experiencias y se refuerzan los elementos considerados como débiles; es decir, el discente deja de ser un receptor pasivo de información proveniente del docente, sino que él a partir de los aprendido comienza a resolver casos prácticos, problemas y tener un pensamiento crítico.

Ahora bien, según lo expresa la Organización de las Naciones Unidas para la Cultura, la Ciencia y la Educación -Unesco(2017), es menester que en el ámbito global se garantice el acceso a la información y se forme recurso humano vinculado al desarrollo económico y social de cada país. En ese propósito, los gobiernos deben contribuir con las instituciones educativas, empresas e industrias de distinta índole para fortalecer las plataformas tecnológicas que permitan la innovación e implementación de nuevos métodos de enseñanza que exploten los recursos de las TIC con el fin de desarrollar capital humano capaz de afrontar retos en su ámbito laboral.

A la luz de las ideas planteadas, en Latinoamérica, son numerosas las instituciones educativas que integran las TIC en los distintos niveles educativos. En el caso particular de México, se observa una práctica efectiva de los modelos emergentes como el aprendizaje invertido (AI), tal como lo expresan Martínez y Esquivel (2018) quienes evaluaron sus efectos en estudiantes de bachillerato, con resultados positivos, ya que se corroboró mediante la examinación, la asimilación adecuada de contenidos y el desarrollo de habilidades de nivel superior en el uso del idioma inglés.

Cabe destacar que la programación de computadoras guarda cierta analogía con el aprendizaje de idiomas, ya que con arreglo a lo expresado por García (2014), esta actividad consiste en escribir un conjunto de 
instrucciones utilizando un lenguaje específico provisto de símbolos, estructuras sintácticas y funciones bien definidas para indicarle al computador que ejecute determinadas tareas orientadas a la solución de algún problema. En tal sentido, esta es una labor que con el transcurrir del tiempo y a la par de los avances tanto de hardware como de software, ha cobrado importancia, pues es necesario formar recurso humano orientado a crear soluciones mediante programas de computadora a fin de asistir al ser humano en sus tareas cotidianas.

A la luz de lo anterior, son numerosas las instituciones educativas de nivel superior que ofrecen carreras relacionadas con el área de tecnología, tal es el caso de Venezuela, específicamente en la Universidad del Zulia, donde existe el Programa de Humanidades y Educación, Mención Informática en el Núcleo Costa Oriental del Lago; el cual tiene como propósito la formación de docentes que a través del uso de estrategias, técnicas, métodos y recursos para el aprendizaje, puedan a su vez, instruir a los estudiantes de los distintos niveles educativos en el uso de las herramientas tecnológicas para desempeñar distintos roles a lo largo de su vida y desarrollar software educativo para apoyar el proceso. De allí la necesidad de utilizar estrategias innovadoras para formar profesionales competentes en el área de programación, ya que forma parte integral del perfil del egresado.

A la luz de los planteamientos anteriores, se pudo percibir a través de una interacción inicial con docentes y estudiantes que debido a la situación contextual del país, donde se dificulta el acceso físico a las instalaciones académicas, se hace necesario, buscar estrategias, métodos y modelos de aprendizaje orientados al aprovechamiento máximo del tiempo dentro del aula e ir más allá, es decir, resulta pertinente colocar a disposición de los discentes un conjunto de recursos para su revisión previa en el hogar a fin de utilizar las clases en aula para construir conocimientos mediante la práctica. En ese sentido, se podrían aplicar al ámbito de la programación dentro del contexto de estudio.

Sobre la base de las ideas anteriores, el presente estudio busca describir los pilares del aprendizaje invertido en virtud de ser aplicado por los docentes de programación del Programa de Humanidades y Educación, mención Informática, de la Universidad del Zulia, Núcleo Costa Oriental del Lago. De esta forma, esta investigación podrá servir como base para otros profesionales de la academia a fin de utilizar este modelo de aprendizaje en su ejercicio docente.

A los efectos de brindar un soporte teórico adecuado al presente estudio, se realizó una revisión de la literatura relacionada con la temática, de los aportes de diversos autores reconocidos, vinculados tanto en las estrategias para el aprendizaje como en programación. Los cuales se presentan a continuación:

\section{El Aprendizaje invertido}

De acuerdo con lo expresado por Bergmann y Sams (2014), el aprendizaje invertido (flipped learning), implementado mediante el aula invertida (flipped classroom) es un modelo de aprendizaje en el cual, se colocan al alcance de los estudiantes contenidos, recursos y materiales multimedia para que sean revisados en sus hogares y luego, se produzca un intercambio activo de conocimientos dentro del aula, siendo ellos los responsables de su aprendizaje de forma autónoma.

Sobre estas ideas, Sedoff (2018) establece que el flipped learning consiste en invertir el proceso de enseñanza-aprendizaje, de manera que lo que tradicionalmente se hacía en el aula, se transfiere fuera de ella y se realiza en casa $u$ otro lugar. De esta manera, cuando se acude al 
aula, el estudiante ya tiene una idea del tema y una guía para trabajar en clase. Su finalidad es aprovechar al máximo el tiempo utilizado para las sesiones presenciales.

Como complemento a lo anterior, Tourón, Santiago y Diez (2014) indican que el aprendizaje inverso (flipped learning) es un enfoque pedagógico en el que la instrucción directa se desplaza del espacio del aprendizaje en grupo al espacio del aprendizaje individual, como resultado de ello, el espacio del grupo se transforma en un ambiente de aprendizaje dinámico e interactivo, en el que el educador guía a los estudiantes mientras estos aplican los conceptos y se implican creativamente en la materia.

A los efectos del presente estudio, se considera el aprendizaje invertido como un modelo emergente que promueve un ambiente interactivo donde el profesor coloca a disposición de los estudiantes, recursos audiovisuales para que éstos los accedan desde su hogar antes de las clases en el aula. De esta manera, se utilizan estos encuentros para aplicar las teorías estudiadas, aclarar dudas y fortalecer la construcción de conocimientos en colectivo. En otras palabras, se deja de lado el paradigma tradicional donde el profesor es el centro y proveedor de la información, mientras los estudiantes son los receptores pasivos.

\section{Pilares del aprendizaje invertido}

Los autores del modelo aprendizaje invertido, Bergman y Sams (2014) plantean lo se ha denominado los cuatro pilares del flipped learning, representados por el acrónimo FLIP. Estos se describen a continuación:

\section{F: Flexible Enviroment o Ambiente} Flexible: Conforme con Calvillo (2015), este pilar hace referencia a la adaptabilidad del entorno de trabajo, ya que el estudiante puede decidir dónde y cuándo aprender mientras que el profesor debe flexibilizar el tiempo necesario para que se produzca el aprendizaje. Igualmente, Bergman y Sams (2014) establecen que el profesor debe permitir una redistribución física del aula, para permitir el trabajo autónomo de cada grupo. Por consiguiente, la flexibilidad no se refiere únicamente al aspecto físico, ya que el docente debe adaptarse a los tiempos de aprendizaje de cada grupo, pues, éstos pueden variar.

Sobre la base de las ideas anteriores, el ambiente flexible se refiere entonces a la adaptabilidad de los espacios de aprendizaje a las necesidades de los estudiantes, considerando también los tiempos de cada uno para la asimilación de los contenidos y la construcción de conocimientos; sin presionar a los actores del proceso, pues, cada uno aprende a su propio ritmo.

Ahora bien, en el caso de la programación se pueden adecuar los espacios físicos para tener una estructura de salón colaborativo (colocando los asientos en forma semicircular), a fin de propiciar la participación y el intercambio de saberes. Esto también puede ser aplicado a los laboratorios de informática, dejando abierta el área central con el fin de facilitar el desplazamiento del docente y la interacción de los estudiantes. Igualmente, se pueden proveer recursos multimedia que versen sobre los fundamentos de determinados lenguajes, tales como estructuras secuenciales, decisivas o repetitivas, con el fin de favorecer la comprensión de los contenidos y homologar en la medida de lo posible, los tiempos de aprendizaje.

L (Learning Culture). Cultura de aprendizaje: Según lo afirma Calvillo (2015), con este pilar, el alumnado se convierte en el centro de atención dentro del aula, donde participan activamente en la formación de su propio conocimiento y se les posibilita su autoevaluación. Por su parte, Tourón y otros 
(2014) indican que el proceso de aprendizaje se centrará en el estudiante, para promover discusiones sobre diversos tópicos de forma más profunda y con mayores oportunidades de aprendizaje. Esto se enmarca en lo establecido por Bergman y Sams (2014), cuando afirman que esto permite un mayor involucramiento del alumno en su propio proceso de aprendizaje. Ahora bien, el rol docente se redefine, pues debe propiciar la participación de todos mediante la retroalimentación constante.

A la luz de las ideas anteriores, se considera entonces que este pilar consiste en centrar la atención en los discentes dentro del aula, con el fin de propiciar el intercambio de ideas y la discusión, como estrategias para profundizar la comprensión de los contenidos. De esta manera, al aplicarse en el ámbito de la programación, se pueden planificar debates o disertaciones sobre las estructuras típicas de los lenguajes de programación, así ellos internalizarán su utilidad e importancia. Igualmente, se pueden plantear ejercicios para su posterior resolución desde distintos puntos de vista, es decir, se evidenciaría la flexibilidad de estas técnicas en la resolución de problemas.

I (Intentional Content). Contenido intencional: Este tercer pilar, como lo refiere Calvillo (2015), está relacionado con la necesidad de decidir qué es lo importante para el alumnado de una materia concreta y cuáles son los materiales con los cuales debería contar para construir su aprendizaje. Esto se realiza con el propósito de optimizar el tiempo de clase a fin de adoptar diversos métodos de enseñanza según el nivel educativo o la materia. En ese orden y dirección, Tourón y otros (2014) establecen un rol del profesor-guía, orientado al entendimiento de los conceptos para lograr una fluidez en el procesamiento. Es así como acorde con cada materia y nivel, se deciden los conceptos que serán abordados en el trabajo personal del alumno y aquellos que deben ser enseñados por el docente.

Como complemento a lo anterior, se puede operacionalizar este pilar como la necesidad de clasificar los temas en función de su naturaleza y complejidad, así el docente podrá determinar cuáles se pueden abordar de forma autónoma por parte de los estudiantes y en contraste, aquellos que deben ser explicados por él. En tal sentido, dentro del ámbito de la programación, aquellos contenidos como la estructura básica de un programa, operadores, uso expresiones e incluso, estructuras iterativas y problemas sencillos, pudieran ser presentadas en recursos multimedia antes de las sesiones de aula, dejando los casos combinados o problemas complejos del lado del profesor.

\section{P (Professional Educator).} Educadores profesionales: El último pilar, en concordancia con el criterio de Calvillo (2015), hace referencia al papel del profesorado en un aula invertida, el cual se fundamenta en observar, guiar y evaluar el trabajo del alumnado en tiempo real, reflexionar sobre su práctica, compartir y aprender de otros docentes o asumir roles menos visibles dentro del aula. Por otra parte, Bergman y Sams (2014) expresan que el docente debe observar constantemente a sus estudiantes con el propósito de proporcionarles una retroalimentación inmediata en virtud de evaluar su trabajo constantemente. También, para Tourón y otros (2014) debe seguir una filosofía reflexiva y mejorar constantemente sus prácticas.

A los efectos del presente estudio, se considera el rol docente como un guíaparticipante del proceso de aprendizaje que, a su vez, tiene la responsabilidad de evaluar al instante, brindando la retroalimentación oportuna a cada estudiante en función de su desempeño e igualmente, debe tener la 
apertura para recibir sugerencias o recomendaciones que le permitan mejorar continuamente. Ahora bien, en el ámbito de la programación, el docente puede proponer ejercicios o casos prácticos a fin de ser resueltos por los alumnos y en ese punto evaluar su desenvolvimiento, proporcionándoles información pertinente sobre los aspectos fuertes y debilidades detectadas.

\section{MÉTODO}

El presente estudio fue de tipo descriptivo y de campo; de conformidad con lo expuesto por Hernández, Fernández y Baptista (2014), quienes aseguran que la investigación de este tipo busca especificar propiedades o características de determinado fenómeno. En tal sentido, se ubica dentro de esta tipología, ya que se identificaron cada una de las características o pilares del aprendizaje invertido dentro del contexto estudiado a partir de sus propiedades o atributos esenciales.

En ese orden de ideas, se clasificó como un estudio de campo, a juzgar por Arias (2012) quien lo presenta como la recolección de datos directamente de la realidad. Por ende, se considera dentro de esta modalidad, ya que la información se recopila en el contexto donde se encuentra el objeto de estudio; es decir, el Programa de Humanidades y Educación, Mención Informática de la Universidad del Zulia, Núcleo Costa Oriental del Lago, ubicado en la Ciudad de Cabimas, los cuales, representan una fuente primaria, sin la intervención de intermediarios o fuentes indirectas.

Así mismo, corresponde con un diseño no experimental - transeccional, en correspondencia con el criterio de Hernández y otros (2014), quienes expresan que en este caso, no se manipulan deliberadamente las variables y se recolectan datos en un solo momento. Es de destacar el presente estudio carece de experimentación, por ende, no existen grupos de control o experimentales donde exista control alguno sobre la variable. Cabe mencionar que la aplicación del instrumento de recolección de datos, se realizó en un solo momento durante el transcurso de la investigación.

A efectos de la presente investigación, se tomó una población finita para la aplicación del instrumento de recolección de datos, la cual estuvo conformada por cinco (05) docentes especialistas en el área de programación y estrategias para el aprendizaje del Programa de Humanidades y Educación Mención Informática del Núcleo Costa Oriental del Lago de la Universidad del Zulia. El instrumento utilizado para la recolección de datos fue el cuestionario. En ese propósito, Arias (2012) plantea que el formato de cuestionario es un instrumento de recolección de información que contiene un conjunto de ítems organizados sistemáticamente para ser respondidos por los sujetos de la investigación.

Con el propósito de medir la variable aprendizaje invertido, se utilizó un cuestionario aplicado a la población descrita anteriormente, el cual estuvo estructurado por un total de 12 proposiciones afirmativas, utilizando una escala de medición tipo Likert, cuyas alternativas son: nunca, casi nunca, algunas veces, casi siempre y siempre, asignándoles valores numéricos del uno al cinco (1-5). El instrumento fue debidamente validado y se estimó su confiabilidad. Acorde con lo expuesto por Hernández y otros (2014), la validez se refiere al grado en que un instrumento mide la variable que pretende medir, mientras la confiabilidad se refiere al grado en que su aplicación repetida al mismo sujeto u objeto produce resultados similares.

Para cumplir con estos criterios, la validez del contenido del instrumento, se determinó mediante la revisión de los ítems 
que lo conforman, a través del juicio de cinco (05) expertos en el área de informática y estrategias para el aprendizaje, quienes revisaron su pertinencia. También, para el cálculo de la de confiabilidad, se aplicó al instrumento a tres (03) sujetos con características similares a la población objeto de estudio

En ese sentido, se calculó del coeficiente de confiabilidad, se utilizó el software estadístico SPSS, versión 23, mediante el coeficiente Alfa de Cronbach, obteniéndose un valor de 0.912, lo cual representa un instrumento altamente confiable, en correspondencia con el baremo presentado por Hernández y otros (2014). Igualmente, para la interpretación de los resultados, se utilizaron cinco categorías, las cuales se observan en el cuadro 1 :

Cuadro 1. Baremo para la interpretación de la media aritmética

\begin{tabular}{cc}
\hline Media & Interpretación \\
\hline $1.00-1.80$ & Muy baja presencia \\
$1.81-2.60$ & Baja presencia \\
$2.61-3.40$ & Moderada presencia \\
$3.41-4.20$ & Alta presencia \\
$4.21-5.00$ & Muy Alta presencia \\
\hline
\end{tabular}

Fuente: Elaboración de los autores

RESULTADOS Y DISCUSIÓN

En este apartado, se presentan las frecuencias relativas en formato porcentual, correspondientes a cuatro pilares del aprendizaje invertido denotando las distintas categorías de respuesta dadas por los sujetos integrantes de la población, así como los promedios obtenidos a cada una de ellas (ver Tabla 1). Esto con el propósito de proporcionar una interpretación adecuada con base en el baremo previamente presentado.

Tabla 1. Resultados obtenidos: Pilares del aprendizaje invertido.

\begin{tabular}{lcccc}
\hline $\begin{array}{c}\text { Pilares del } \\
\text { aprendizaje invertido }\end{array}$ & $\begin{array}{c}\text { Ambiente } \\
\text { flexible } \\
\text { fr\% } \%\end{array}$ & $\begin{array}{c}\text { Cultura de } \\
\text { aprendizaje } \\
\text { fr\% }\end{array}$ & $\begin{array}{c}\text { Contenido } \\
\text { intencional } \\
\text { fr\% }\end{array}$ & $\begin{array}{c}\text { Educadores } \\
\text { profesionales } \\
\text { fr\% }\end{array}$ \\
\hline Siempre & - & 6,67 & 13,33 & - \\
Casi siempre & 13,33 & 6,67 & 13,33 & 20,00 \\
Algunas veces & 20,00 & 26,67 & 26,67 & 13,33 \\
Casi Nunca & 26,67 & 13,33 & 20,00 & 26,67 \\
Nunca & 40,00 & 46,67 & 26,67 & 40,00 \\
Promedio aritmético & 2,07 & 2,13 & 2,67 & 2,13 \\
Interpretación del & Baja presencia & Baja presencia & Moderada presencia & Baja presencia \\
promedio aritmético & &
\end{tabular}

Fuente: Elaboración de los autores partir de los cálculos arrojados por SPSS (2019) 
Al observar el comportamiento del pilar ambiente flexible, se obtuvo un $40 \%$ de respuestas en la opción nunca, mientras que las alternativas casi nunca y algunas veces se ubicaron en un $26,67 \%$ y $20 \%$ respectivamente, dejando sólo un $13,33 \%$ de los sujetos en la categoría casi siempre. Por otra parte, la media reportó un valor de 2,07, ubicándose en una baja presencia conforme con el baremo de interpretación.

Estos resultados se traducen en una tendencia a no propiciar un ambiente adaptable para los discentes, manejando la distribución física del aula de acuerdo con los parámetros tradicionales, sin mayores cambios y tampoco se consideran a profundidad la importancia del aprendizaje autónomo, por lo cual, se infiere que los docentes se mantienen como el eje de la clase, con apoyo de recursos didácticos, pero no los colocan a disposición de los estudiantes antes de los encuentros áulicos.

Lo anterior puede deberse a fluctuaciones del servicio de internet y fluido eléctrico. Esto se contrapone a los postulados de Bergman y Sams (2014), quienes enfatizan en la necesidad de redistribuir físicamente el salón de clases en función de promover la participación y el intercambio de saberes, al punto que se consideran los tiempos de aprendizaje de cada grupo, ya que pueden ser distintos entre sí. Igualmente, se oponen a lo establecido por Calvillo (2015) quien otorga relevancia a la capacidad del docente para adaptar los recursos a fin de colocarlos al alcance de los alumnos para que estos puedan aprender a su propio ritmo.

Como complemento a lo anterior, se puede crear un ambiente flexible en las clases de programación, promoviendo la discusión de los distintos temas entre los estudiantes mediante un salón colaborativo, distribuido de forma semicircular. El docente a su vez, colocará recursos pertinentes en la web, donde se aborden los fundamentos de los lenguajes, operadores típicos, estructura general de los programas, palabras reservadas, ciclos $\mathrm{y}$ decisiones para que cada estudiante pueda estudiarlos a su tiempo antes de las clases en el aula.

En cuanto al segundo pilar, denominado cultura de aprendizaje, se pudo observar que un $46,67 \%$ de las respuestas se ubicaron en la opción nunca, siendo el mayor puntaje. De esta forma, las opciones casi nunca y algunas veces reportaron un $13,33 \%$ y $26,67 \%$ respectivamente, mientras las categorías casi siempre y siempre relejaron un $6,67 \%$ cada una. En ese sentido, se denota una inclinación a la no existencia de una cultura de aprendizaje. Por consiguiente, la media aritmética de este tópico fue de 2,13 significando una baja presencia, lo cual, ratifica los resultados porcentuales.

Esto quiere decir que no se considera a los estudiantes como el eje central del proceso de aprendizaje ni tampoco como agentes activos. Esto difiere de lo expresado por Calvillo (2015), quien hace énfasis en el rol del discente como protagonista y responsable de su propia formación, siendo críticos al punto de estar en capacidad de realizar su autoevaluación.

A la luz de los resultados obtenidos, se puede establecer que es necesario reforzar la cultura de aprendizaje en el marco del flipped learning, dentro del contexto estudiado. Por ende, en el ámbito de la programación, se pueden diseñar estrategias fuera del aula (online) como la resolución de problemas sencillos de forma individual, mediante un compilador de determinado lenguaje de programación para luego, ser socializados dentro del aula, discutiendo a su vez los resultados y distintas posibilidades de resolución e incluso, plantear casos para su resolución en grupos. De esta manera se sembrarán las bases del pensamiento crítico y multimodal en el discente, proporcionándole 
herramientas para responsabilizarse de su aprendizaje.

Ahora bien, en cuanto al pilar llamado contenido intencional, se pudieron observar puntajes bastantes parejos, siendo $26,67 \%$ en las opciones nunca y algunas veces, 13,33\% para las alternativas siempre y casi siempre, mientras que se reportó un $20 \%$ el valor obtenido en la categoría casi nunca. Además, la media de 2,67 se encuentra ubicada en el rango de moderada presencia, lo cual, significa que los docentes se preocupan de manera relativa por el diseño de materiales didácticos para que los estudiantes los estudien de manera autónoma y por otra parte, también están conscientes de aquellos contenidos a ser explicados propiamente por el docente. Esto coincide con lo expresado por Calvillo (2015), quien expresa la necesidad del docente por clasificar el material, dejando los contenidos más sencillos como objeto de estudio autónomo y los más complejos para ser desarrollados por éste.

A la luz de los hallazgos obtenidos, se puede afirmar que en lo concerniente al contenido intencional, los docentes del área de programación están familiarizados con el uso de las tecnologías y conocen ampliamente los contenidos impartidos, por lo tanto, pueden seleccionar los temas teóricos, estructuras básicas de los programas, palabras reservadas, operadores, expresiones y otros conceptos fundamentales para diseñar recursos a fin de ser estudiados por los discentes en sus hogares de forma autónoma y tratar los temas vinculados con los ciclos, decisiones, arreglos, interfaces gráficas e implementaciones más avanzadas dentro del aula. Sin embargo, todo dependerá de las habilidades previamente adquiridas por los estudiantes, pues, existen lenguajes de programación muy intuitivos, con ayudas en línea robustas, dotadas de ejemplos y casos de aplicación.
Por último, el cuarto pilar denominado educadores profesionales, reportó un $40 \%$ en la opción nunca, así como un 26,67\%, 13,33\% y $20 \%$ en las alternativas casi nunca, algunas veces y casi siempre, respectivamente. Por su parte, el promedio fue de 2,13 representando una baja presencia, lo cual denota una debilidad en los docentes, pues, pudiesen no estar retroalimentando oportunamente o en tiempo real a los estudiantes acerca de los aspectos a mejorar, permitiéndoles reflexionar sobre sus avances y logros.

En ese sentido, los profesores manifiestan estar orientados a mejorar continuamente, pero no cuentan con recursos suficientes para la actualización de conocimientos. Esto dista de los postulados de Bergman y Sams (2014), Tourón y otros (2014) y Calvillo (2015), pues, convergen en la responsabilidad del docente de brindar el feedback a los estudiantes al momento de observar su desempeño para proveerles herramientas de reflexión. Del mismo modo, deben estar dispuestos a recibir sugerencias a efectos de optimizar su labor en beneficio del colectivo estudiantil.

Ahora bien, en el ámbito de la programación, es importante que los docentes mantengan una mentalidad abierta, ya que un problema similar puede tener distintas alternativas de respuesta y los discentes pueden presentar diversidad de soluciones ante el mismo caso. En tal sentido, se fomenta el pensamiento crítico-reflexivo a la par del uso instrumental de los lenguajes de programación dentro del contexto donde se desenvolverán profesionalmente.

\section{CONCLUSIONES}

A través de la presente investigación, se pudieron describir los cuatro pilares del aprendizaje invertido (flipped learning) en los docentes de programación, de la Universidad 
del Zulia, Núcleo Costa Oriental del Lago, específicamente adscritos al Programa de Humanidades y Educación, Mención Informática, es decir, ambiente flexible, cultura de aprendizaje, contenido intencional y educadores profesionales, obteniéndose mayormente una baja presencia dentro del contexto, lo cual, significa que se deben reforzar la difusión de este modelo de aprendizaje a fin de presentar a los profesores, una alternativa para dinamizar las clases y romper los límites del aula, aprovechando incluso el tiempo en el hogar.

En lo que respecta a la creación de un ambiente flexible, los docentes deben preocuparse de proveer los recursos necesarios para que los estudiantes asimilen los contenidos a su propio ritmo, contando con el tiempo suficiente antes de las clases presenciales. En ese propósito, se deben crear materiales interactivos y dinámicos en función de incentivar el aprendizaje autónomo, consolidando las bases requeridas para afrontar la construcción de conocimientos durante las sesiones en el aula. Así, los profesores de programación pueden crear videos, presentaciones electrónicas, animaciones e interactividades con los contenidos fundamentales sobre los lenguajes de programación para que los discentes los comprendan antes de abordar la solución de problemas o temáticas más complejas.

En otro orden de ideas, los docentes deben promover una cultura de aprendizaje donde el estudiante sea el centro de la actividad dentro y fuera del aula, es decir, procurará aplicar estrategias dirigidas a promover la discusión grupal de los contenidos tratados con la finalidad de formar un pensamiento crítico sobre la utilidad e importancia práctica de lo aprendido y su implementación dentro de su entorno. Ahora bien, los docentes del área de programación pueden proponer casos de análisis o ejercicios para que los discentes de manera individual o colectiva, aporten sus soluciones, las cuales, a su vez, pueden ser socializadas e intercambiadas, al punto de apropiarse del nuevo conocimiento y desarrollar competencias en esta rama de la informática.

Ahora bien, en cuanto al tercer pilar, denominado contenido intencional, se puede indicar que los docentes deben procurar la clasificación de los contenidos con el fin de separar los que serán estudiados de forma autónoma por los estudiantes y por el contrario, aquellos dirigidos por el docente. Igualmente, deberán determinar cuáles son las mejores estrategias o recursos en función de la naturaleza y su complejidad para consolidar el proceso de aprendizaje. Entonces, los profesores de programación, deben tener especial cuidado con la categorización de los contenidos tratados, colocando materiales accesibles por los estudiantes cuya complejidad no exceda las capacidades iniciales de sus alumnos y a su vez, dejar los ejercicios o problemas más complejos para las clases dentro del aula.

Por último, el pilar llamado educadores profesionales, está relacionado con promover la mejora continua en los docentes, los cuales deben mantener un amplio criterio al momento de evaluar y proporcionar la retroalimentación necesaria para crear conciencia en los estudiantes acerca de sus fortalezas y debilidades. Igualmente, tienen el deber de actualizarse en conocimientos para mantenerse vigentes y formar recurso humano pertinente con su entorno laboral.

A la luz de todo lo expuesto anteriormente, se puede concluir que los docentes de programación poseen los prerrequisitos necesarios para la implementación del aprendizaje invertido en sus unidades curriculares, pues, sólo falta reforzar los 
conocimientos necesarios en lo referente a este modelo de enseñanza, pues ya están en la capacidad de diseñar recursos multimedia, utilizar estrategias de construcción de conocimientos y están familiarizados con los contenidos que imparten. En ese sentido, se pueden coordinar esfuerzos institucionales para adoptar el flipped learning como una alternativa para la formación de nuevos profesionales en el Programa de Humanidades y Educación, Mención Informática del Núcleo Costa Oriental del Lago de la Universidad del Zulia.

\section{REFERENCIAS}

Arias, F. (2012). El Proyecto de Investigación. Introducción a la Metodología Científica (6ạ. Edición). Caracas: Editorial Epísteme

Bergman, J. y Sams, A. (2014). Dale la vuelta a tu clase: Lleva tu clase a cada estudiante, en cualquier momento y cualquier lugar. ( $2^{\mathrm{a}}$ ed.). Madrid: Santa María Ediciones

Calvillo, A. (2015). Los 4 pilares y los 11 indicadores del Flipped Learning. Recuperado de http://www.musikawa.es/media/los-4- pilares-y-los-11-indicadores-del-flippedlearning-autoevaluate-flippedkawaflippedclassroom/

García, B. (2014). Fundamentos de programación. Lulu Press Incorporated.

Hernández, R. Fernández, C., y Baptista, M. (2014). Metodología de la Investigación (6ª Edición). Editorial Mc. Graw Hill

Martínez, W. y Esquivel, I. (2018). Uso del modelo de aprendizaje invertido en un bachillerato público. Revista de Educación a Distancia. Número 58, Artículo 11

Sedoff, M. (2018). Claves para aplicar el flipped learning. Recuperado de http://eduprensa.com/miguel-sedoffclaves-aplicar-flipped-learning/

Tourón, J., Santiago, R. y Diez, A. (2014). The flipped classroom. Cómo convertir la escuela en un espacio de aprendizaje. Barcelona: Digital-Text. Grupo Oceano

Unesco. (2017) Competencias y estándares TIC desde la dimensión pedagógica: Una perspectiva desde los niveles de apropiación de las TIC en la práctica educativa docente ISBN: 2016, Pontificia Universidad Javeriana - Cali 